\title{
ANALYSIS OF PARALLELOGRAM MECHANISM USED TO PRESERVE REMOTE CENTER OF MOTION FOR SURGICAL TELEMANIPULATOR
}

\author{
R. TROCHIMCZUK \\ Department of Automatic Control and Robotics \\ Faculty of Mechanical Engineering \\ Bialystok University of Technology, POLAND \\ E-mail: r.trochimczuk@pb.edu.pl
}

\begin{abstract}
This paper presents an analysis of a parallelogram mechanism commonly used to provide a kinematic remote center of motion in surgical telemanipulators. Selected types of parallel manipulator designs, encountered in commercial and laboratory-made designs described in the medical robotics literature, will serve as the research material. Among other things, computer simulations in the ANSYS 13.0 CAD/CAE software environment, employing the finite element method, will be used. The kinematics of the solution of manipulator with the parallelogram mechanism will be determined in order to provide a more complete description. These results will form the basis for the decision regarding the possibility of applying a parallelogram mechanism in an original prototype of a telemanipulator arm.
\end{abstract}

Key words: parallelogram mechanism, surgical telemanipulator, robot minimally invasive surgery, stiffness FEM analysis, RCM mechanism.

\section{Introduction}

Surgical telemanipulators are introduced into hospitals' operating rooms with increasing frequency. They constitute specialized technical equipment providing a modern surgeon with even better precision and control over performed actions, stability of instrument movement, while simultaneously eliminating fatigue and physiological effects, e.g., shaking of limbs due to holding of an instrument in a given position for a long time. Surgical telemanipulators also make it possible to provide the proper sterility for a procedure, isolating the surgeon from the effects of, e.g., X-ray radiation, and they reduce the risk of potential infections resulting from contact with the patient. Today, surgical telemanipulators are mainly used to perform open surgical procedure and operations as well as laparoscopic procedures. In the case of procedures of the first type, designing of a telemanipulator's kinematics is subject to a relatively lower number of technical constraints. The design of such a robot's arms is rather frequently similar to typical solutions of industrial manipulators with 5 or 6 degrees of freedom, with a relatively large workspace and collision space. The other group among the aforementioned procedures is characterized by the insertion of laparoscopic instruments through small incisions in the patient's skin. Due to limited space and the lack of direct visual contact, operating a surgical instrument significantly changes the engineering approach to the design of the structure and method of controlling the telemanipulator's arm. For a procedure of this type to be possible, it is necessary to ensure a so-called remote center of motion (RCM), i.e., the condition necessary for the instrument to pass through one specific point in the patient's body and rotate around intersecting axes. The establishment of a fixed remote center of motion makes it possible to minimize the risk of collision between the patient's body and the robot's arm, thus providing a sufficient movement range for surgical instruments fastened to the telemanipulator's arm. In addition, an RCM provides a relatively simple solution of the forward and inverse kinematics of the surgical telemanipulator, making it possible to create position control for the end effector. In designing practice, a remote center of motion is provided by three methods: 1) through passive point (RCM) fixation, 2) kinematic point fixation and 3) active point fixation (mechatronic) [1]. 
This paper presents an analysis of a parallel manipulator mechanism commonly used to provide a kinematic remote center of motion in surgical telemanipulators. Selected types of parallel manipulator designs, encountered in commercial and laboratory-made designs described in the literature on medical robotics, will serve as the research material. Solid models have been designed by the author for the given structures in the SolidWorks 2015/2016 software environment. Among other things, computer simulations in the ANSYS 13.0 $\mathrm{CAD} / \mathrm{CAE}$ software environment, employing the finite element method, will be used. The kinematics of the solution with the parallel manipulator will be determined in order to provide a more complete description. These results will form the basis for the decision regarding the possibility of applying a parallel manipulator mechanism in an original prototype of a telemanipulator arm that the author of this paper is currently working on at the Faculty of Mechanical Engineering of Bialystok University of Technology.

\section{Description of kinematic designs of manipulators with a parallelogram mechanism adopted for the purpose of research}

Minimally invasive procedures utilizing laparoscopic techniques require the insertion of instruments into the human body through small incisions, or in the case of newer techniques, through natural orifices of the body. Regardless of the instruments used (e.g., endoscopic camera, graspers, coagulators, etc.), what remains constant throughout the entire surgical procedure is, in this case, the opening through which an instrument is inserted (fixed remote center of motion - a kind of ball joint with a diameter up to $15 \mathrm{~mm}$ [2]). For movement of the surgical instrument to be possible, special kinematic designs of the manipulator positioning of the surgical instrument are applied. Spherical manipulator designs with a fixed RCM, unchanging regardless of the position assumed by the manipulator, are one of the most common solutions. Here, the fixed RCM is provided by a manipulator with a parallelogram, most often via kinematic means. From a practical point of view, this rather extensive and complicated geometric structure requires high accuracy of manufacturing and final assembly so that geometric conditions required for an RCM to exist are met. Nevertheless, it allows a significant simplification of the method by which the telemanipulator is controlled by limiting the number of drives and sensor systems.

An analysis of the results of research conducted by various scientific centers in Poland and around the world [1-3, 4-7] shows that designs of spherical telemanipulators, with a parallelogram manipulation mechanism making it possible to meet the kinematic RCM condition, can be presented in a classification as in Fig.1.

The example in Fig.1a is the most classical solution, with a four-bar linkage. It is frequently encountered in industrial robot designs, where it serves to increase rigidity. It was used in one of the works of the author of [4]. The author of this paper used a modification of the design in example b) to design an original arm of a surgical telemanipulator [5]. The design of the mechanism presented in Fig.1c corresponds to [6]. The examples presented in Fig.1d and Fig.1e correspond to designs used in modifications of the Polish RobInHeart cardiac surgery robot [1-3].

a)

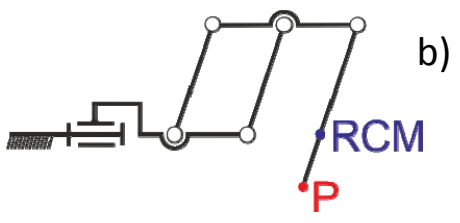

d)

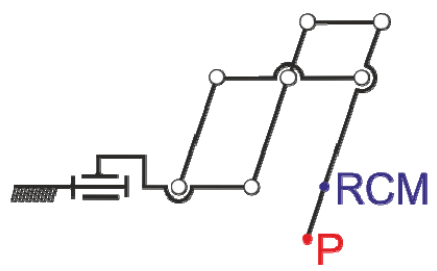

b)

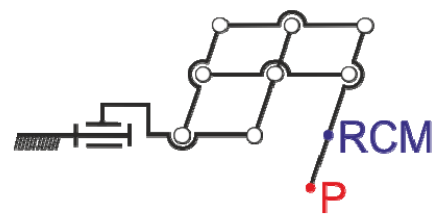

c)

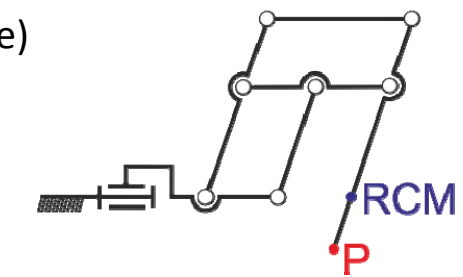

Fig.1. View of mechanism's structures in: a) configuration I; b) configuration II; c) configuration III, d) configuration IV; e) configuration V. 
Solid models of manipulators (Fig.2) were created on the basis of the kinematic structures in Fig.1. Models were not optimized in terms of selection of design parameters, efforts were only made to preserve the mechanism's kinematic structure when designing them. The members of each designed manipulator were created from a circular pipe (the same dimension was selected for every cross-section in five studied configurations, i.e., the lower main member $-12 \mathrm{~mm}$, arms of the four-bar linkage $-7 \mathrm{~mm}$, upper main member $-10 \mathrm{~mm}$, additional connecting members $-5 \mathrm{~mm}$ ). The positions of the four-bar linkage arms were modified by modeling the manipulator's structure (Fig.1), based on placing the arms on opposite sides of the main members - upper and lower. This modification makes it possible to increase the manipulator's movement range compared to the case where the linkage arms were fastened on one side. In addition, the model is devoid of any chamfers and rounding, specially for the purpose of numerical analysis.

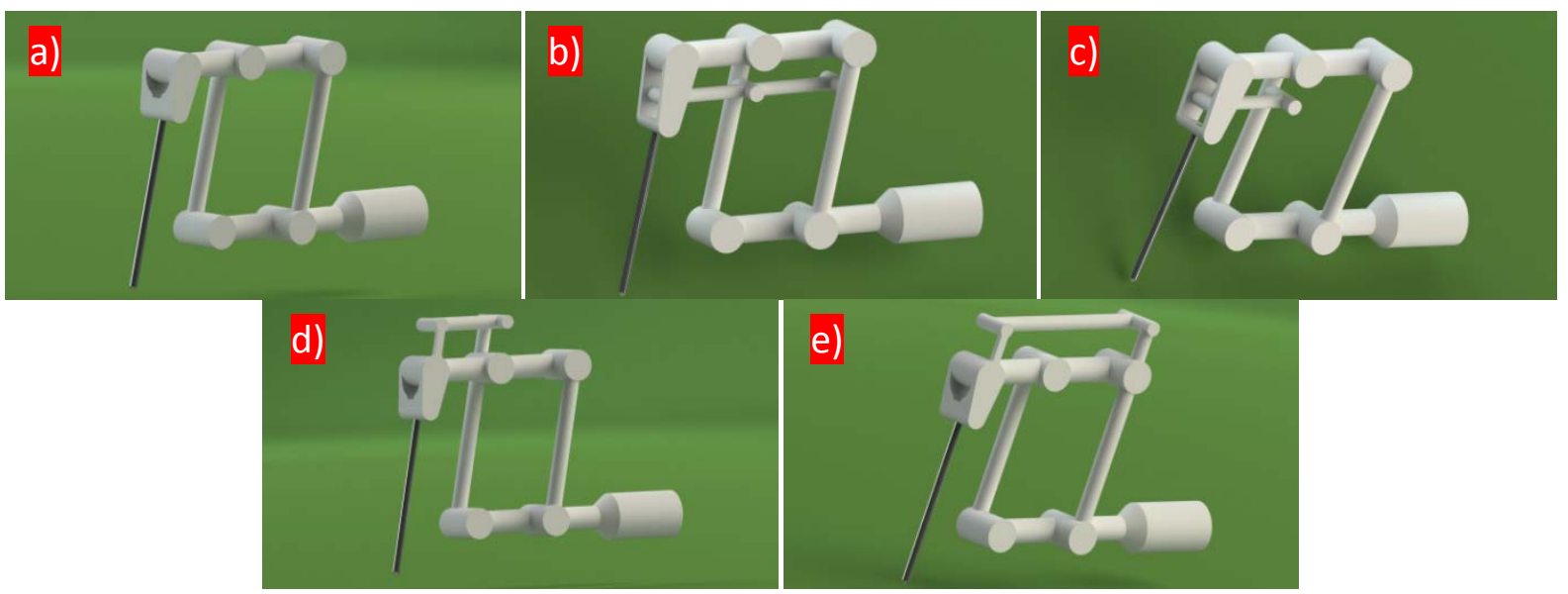

Fig.2. View of mechanism's computational model design in SolidWorks: a) configuration I; b) configuration II; c) configuration III, d) configuration IV; e) configuration V.

Data concerning weights of members and entire manipulator structures along with the parallelogram mechanism, for the material adopted for the study, are given in table 1 (see Tab.3).

Models, created in SolidWorks 2015/2016, were exported to the ANSYS 13.0 Workbench environment, and there they underwent further numerical analysis according to the finite element method by means of ANSYS Static Structural Toolbox.

Table 1. Mass of links in selected configuration of the parallelogram mechanism.

\begin{tabular}{|l|c|c|c|c|c|}
\cline { 2 - 6 } \multicolumn{1}{c|}{} & Config. I & Config. II & Config. III & Config. IV & Config. V \\
\hline Total Mass [kg] & $\mathbf{5 1 . 7 8 3}$ & $\mathbf{5 3 . 1 2 2}$ & $\mathbf{5 2 . 1}$ & $\mathbf{5 4 . 6 2 4}$ & $\mathbf{5 5 . 5 7 8}$ \\
\hline Mass of link 1 [kg] & 7.5477 & 7.5477 & 7.5477 & 7.5477 & 7.5477 \\
\hline Mass of link 2 [kg] & 18.075 & 18.075 & 18.075 & 18.075 & 18.075 \\
\hline Mass of link 3 [kg] & 4.687 & 4.694 & 4.687 & 4.687 & 4.687 \\
\hline Mass of link 4 [kg] & 4.687 & 4.694 & 4.695 & 5.195 & 5.195 \\
\hline Mass of link 5 [kg] & 11.415 & 11.415 & 11.415 & 11.415 & 11.415 \\
\hline Mass of link 6 [kg] & 4.2878 & 2.2123 & 1.2639 & 4.5317 & 3.043 \\
\hline Mass of link 7 [kg] & 1.0842 & 3.4287 & 3.4287 & 2.0885 & 4.5317 \\
\hline Mass of link 8 [kg] & --- & 0.9231 & 0.9231 & 1.0842 & 1.0842 \\
\hline
\end{tabular}




\section{Kinematics of parallelogram mechanism}

The forward kinematics of the parallelogram mechanism are described below. A manipulator in the basic configuration, in which the remote center of motion is provided mechatronically (so-called active remote center of motion), is not kinematically. The procedure described is analogous with respect to other manipulator configurations defined in the paper.

To describe the forward kinematics task the D-H notation given by Craig [8] is used. The general form of the matrix is as follows

$$
{ }_{1}^{i-1} T=\left[\begin{array}{cccc}
\cos \theta_{i} & -\sin \theta_{i} & 0 & a_{i-1} \\
\sin \theta_{i} \cos \alpha_{i-1} & \cos \theta_{i} \cos \alpha_{i-1} & -\sin \alpha_{i-1} & -\sin \alpha_{i-1} d_{i} \\
\sin \theta_{i} \sin \alpha_{i-1} & \cos \theta_{i} \sin \alpha_{i-1} & \cos \alpha_{i-1} & \cos \alpha_{i-1} d_{i} \\
0 & 0 & 0 & 1
\end{array}\right] .
$$

D-H parameters adopted for the parallelogram mechanism are shown in Tab.2 and Fig.3.

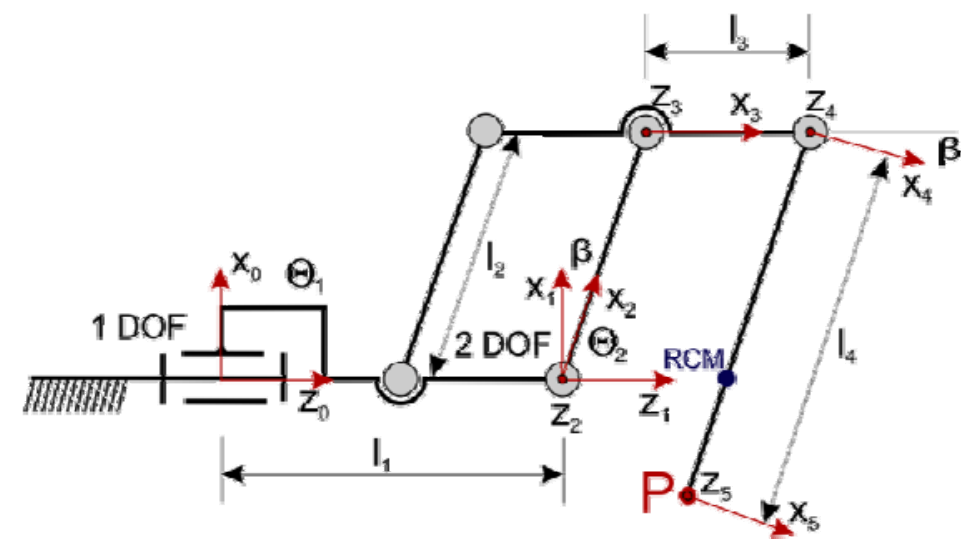

Fig.3. View of D-H parameters of the parallelogram mechanism.

Table 2. D-H parameters of the parallelogram mechanism.

\begin{tabular}{|c|c|c|c|c|}
\hline \multicolumn{5}{|c|}{ D-H parameters } \\
\hline $\boldsymbol{i}$ & $\boldsymbol{\alpha}_{\mathrm{i}-1}$ & $\boldsymbol{a}_{\boldsymbol{i}-1}$ & $\boldsymbol{\Theta}_{\mathbf{i}}$ & $\boldsymbol{d}_{\boldsymbol{i}}$ \\
\hline $0-1$ & 0 & 0 & $\Theta_{1}$ & $l_{1}$ \\
\hline $1-2$ & $-90^{0}$ & 0 & $\Theta_{2}+\beta$ & 0 \\
\hline $2-3$ & 0 & $l_{2}$ & $\begin{array}{c}\Theta_{3}=90^{0}- \\
\left(\Theta_{2}+\beta\right)\end{array}$ & 0 \\
\hline $3-4$ & 0 & $l_{3}$ & $\Theta_{4}=-\beta$ & 0 \\
\hline $4-5$ & 0 & $l_{4}$ & 0 & 0 \\
\hline
\end{tabular}

The transformation matrix according to the accepted parameters for the parallelogram mechanism takes the form 


$$
{ }_{p}^{0} T={ }_{1}^{0} T \cdot{ }_{2}^{1} T \cdot{ }_{3}^{2} T \cdot{ }_{4}^{3} T \cdot{ }_{5}^{4} T .
$$

After substitution we obtain

$$
{ }_{p}^{0} T=\left[\begin{array}{cccc}
A_{11} & A_{21} & -s \theta_{1} & A_{41} \\
A_{12} & A_{22} & 0 & A_{42} \\
A_{13} & A_{23} & 0 & A_{43} \\
0 & 0 & 0 & 1
\end{array}\right]
$$

where

$$
\begin{aligned}
& c \theta_{i}=\cos \theta_{i}, \\
& s \theta_{i}=\sin \theta_{i}, \\
& A_{11}=c\left(\beta+\theta_{2}\right) c \theta_{1} c \theta_{3} c \theta_{4}-c\left(\beta+\theta_{2}\right) c \theta_{1} s \theta_{3} s \theta_{4}, \\
& A_{12}=c\left(\beta+\theta_{2}\right) c \theta_{3} c \theta_{4} s \theta_{1}-c\left(\beta+\theta_{2}\right) s \theta_{1} s \theta_{3} s \theta_{4}, \\
& A_{13}=s\left(\beta+\theta_{2}\right) s \theta_{3} s \theta_{4}-s\left(\beta+\theta_{2}\right) c \theta_{3} c \theta_{4}, \\
& A_{21}=-c\left(\beta+\theta_{2}\right) c \theta_{1} c \theta_{3} s \theta_{4}-c\left(\beta+\theta_{2}\right) c \theta_{1} c \theta_{4} s \theta_{3}, \\
& A_{22}=-c\left(\beta+\theta_{2}\right) c \theta_{3} s \theta_{1} s \theta_{4}-c\left(\beta+\theta_{2}\right) c \theta_{4} s \theta_{1} s \theta_{3}, \\
& A_{23}=s\left(\beta+\theta_{2}\right) c \theta_{3} s \theta_{4}+s\left(\beta+\theta_{2}\right) c \theta_{4} s \theta_{3}, \\
& A_{41}=\left(c\left(\beta+\theta_{2}\right) c \theta_{1} c \theta_{3} c \theta_{4}-c\left(\beta+\theta_{2}\right) c \theta_{1} s \theta_{3} s \theta_{4}\right) l_{4}+ \\
& +c\left(\beta+\theta_{2}\right) c \theta_{1} l_{2}+c\left(\beta+\theta_{2}\right) c \theta_{1} c \theta_{3} l_{3}, \\
& A_{42}=\left(c\left(\beta+\theta_{2}\right) c \theta_{3} c \theta_{4}-s\left(\beta+\theta_{2}\right) s \theta_{3} s \theta_{4}\right) l_{4}-s\left(\beta+\theta_{2}\right) c \theta_{3} l_{3}-s\left(\beta+\theta_{2}\right) l_{2}, \\
& A_{43}=l_{1}-\left(s\left(\beta+\theta_{2}\right) c \theta_{3} c \theta_{4}-s\left(\beta+\theta_{2}\right) s \theta_{3} s \theta_{4}\right) l_{4}-s\left(\beta+\theta_{2}\right) c \theta_{3} l_{3}-s\left(\beta+\theta_{2}\right) l_{2},
\end{aligned}
$$




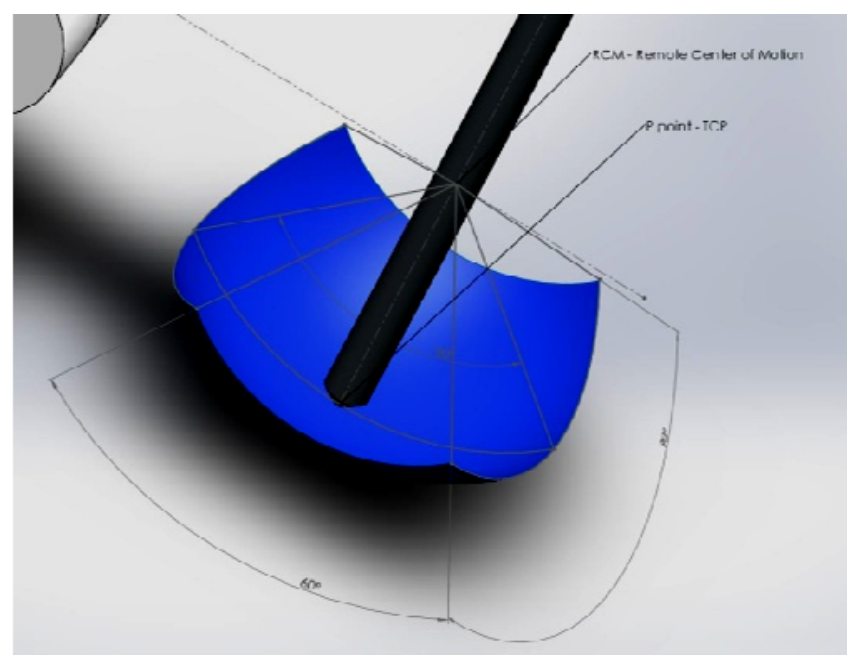

Fig.4. View of working area of parallelogram mechanism.

Knowledge of the workspace within which the end effector - the tip of the surgical instrument, can operate is a significant factor conditioning the use of a telemanipulator with a parallel manipulator mechanism for the purposes of laparoscopic procedures. A surface depicting the workspace (Fig.4) was created in the SolidWorks 2015/2016 software environment on the basis of the geometric values of the telemanipulator's design (regardless of the general structure of the parallel manipulator mechanism) and under the assumption of possible ranges of angles of rotation (from $-45^{0}$ to $+45^{\circ}$ ) for two drives ( 1 and 2 DOF) moving the entire structure.

In an actual structure, the telemanipulator's arm should also be equipped with travel of the surgical instrument to a given depth inside the patient's body, to make it possible for medical personnel to remove the instrument from under the patient's skin. Then, the telemanipulator's workspace will take on the form of a solid bounded from one side by the surface that was created, and by the RCM on the other side.

\section{Assumptions adopted for the purpose of numerical analysis in ANSYS 13.0 workbench software}

A single material from the ANSYS Workbench library - stainless steel, was applied for every member of modeled structure for the purpose of numerical simulations. This material could also be used in biomedical structures. Its properties are defined in Tab.3.

Table 3. Stainless steel material properties used in FEA.

\begin{tabular}{|l|l|}
\hline Density & $7.75 e-006 \mathrm{~kg} \mathrm{~mm}^{\wedge}-3$ \\
\hline Coefficient of Thermal Expansion & $1.7 e-005 \mathrm{C}^{\wedge}-1$ \\
\hline Specific Heat & $4.8 e+005 \mathrm{~mJ} \mathrm{~kg}^{\wedge}-1 \mathrm{C}^{\wedge}-1$ \\
\hline Thermal Conductivity & $1.51 e-002 \mathrm{~W} \mathrm{~mm}^{\wedge}-1 \mathrm{C}^{\wedge}-1$ \\
\hline Compressive Yield Strength & $207 \mathrm{MPa}$ \\
\hline Tensile Yield Strenght & $207 \mathrm{MPa}$ \\
\hline Tensile Ultimate Strength & $586 \mathrm{MPa}$ \\
\hline Young's Modulus & $1.93 e+005 \mathrm{MPa}$ \\
\hline Poisson's Ratio & 0.31 \\
\hline Bulk Modulus & $1.693 e+005 \mathrm{MPa}$ \\
\hline Shear Modulus & 73664 \\
\hline
\end{tabular}


Each of the five configurations of manipulators accepted for testing was subjected to the action of force of the value $(50 \mathrm{~N}$ and $-50 \mathrm{~N})$ that would act on the end effector. The value of $50 \mathrm{~N}$ meant the action of force according to the direction in which gravity acts (in the case of the surgical telemanipulator, this can occur during removal of parts of tissue or organs and their extraction from the patient's body). The value of $50 \mathrm{~N}$ meant that force was applied in the direction reverse to the action of gravity (in the case of the surgical manipulator, this may occur during cutting of the patient's tissue). In addition, Standard Earth Gravity also acted on manipulators $-9806.6 \mathrm{~mm} / \mathrm{s}^{2}$. The following were defined in each manipulator: Contact type as Bonded - automatic and Joints - revolte and fixed. Fixed support definition on 1 face on link 1. Besides Global Coordinate System is additionally defined by Tool Coordinate System associated with TCP-point of end-effector.

Tests were conducted for six cases of selected, characteristic positions of members in a given manipulator configuration (see Fig.5): 1) Normal 90 degree - arms of the parallel manipulator are positioned at a 90 degree angle relative to one another; 2) 45 degree - in this configuration, the linkage's arms are inclined at an angle of 45 degrees relative to the lower main member, 3) 135 degree - in this configuration, the linkage's arms are positioned at an angle of 135 degrees relative to the lower main member; 4) Normal 90 degree/45 side - arms of the linkage positioned as in the case of Normal 90 degree and also inclined at 45 degrees relative to the first member; 5) 45 degree/45 side - the linkage's arms are positioned as in the 45 degree case and also inclined at 45 degrees relative to the first member; 6) 135 degree/45 side - the linkage's arms are positioned as in the 135 degree case and also inclined at 45 degrees relative to the first member. Both bending and torsion forces act in the studied cases due to the geometry adopted for the manipulators.

In order to generate the finite element mesh for all links of the Automatic Method and Mapped Face Meshing on selected faces was use (example Fig.6). In configuration I we used: 21155 nodes in study 1; 21588 nodes in case study 2; 21363 nodes in case study 3; 21385 nodes in case study 4; 21423 nodes in case study 5; 21587 nodes in case study 6 and appropriately 9103 elements in case study 1; 9334 elements in case study 2; 9196 elements in case study 3; 9204 elements in case study 4; 9255 elements in case study 5; 9330 elements in case study 6 .
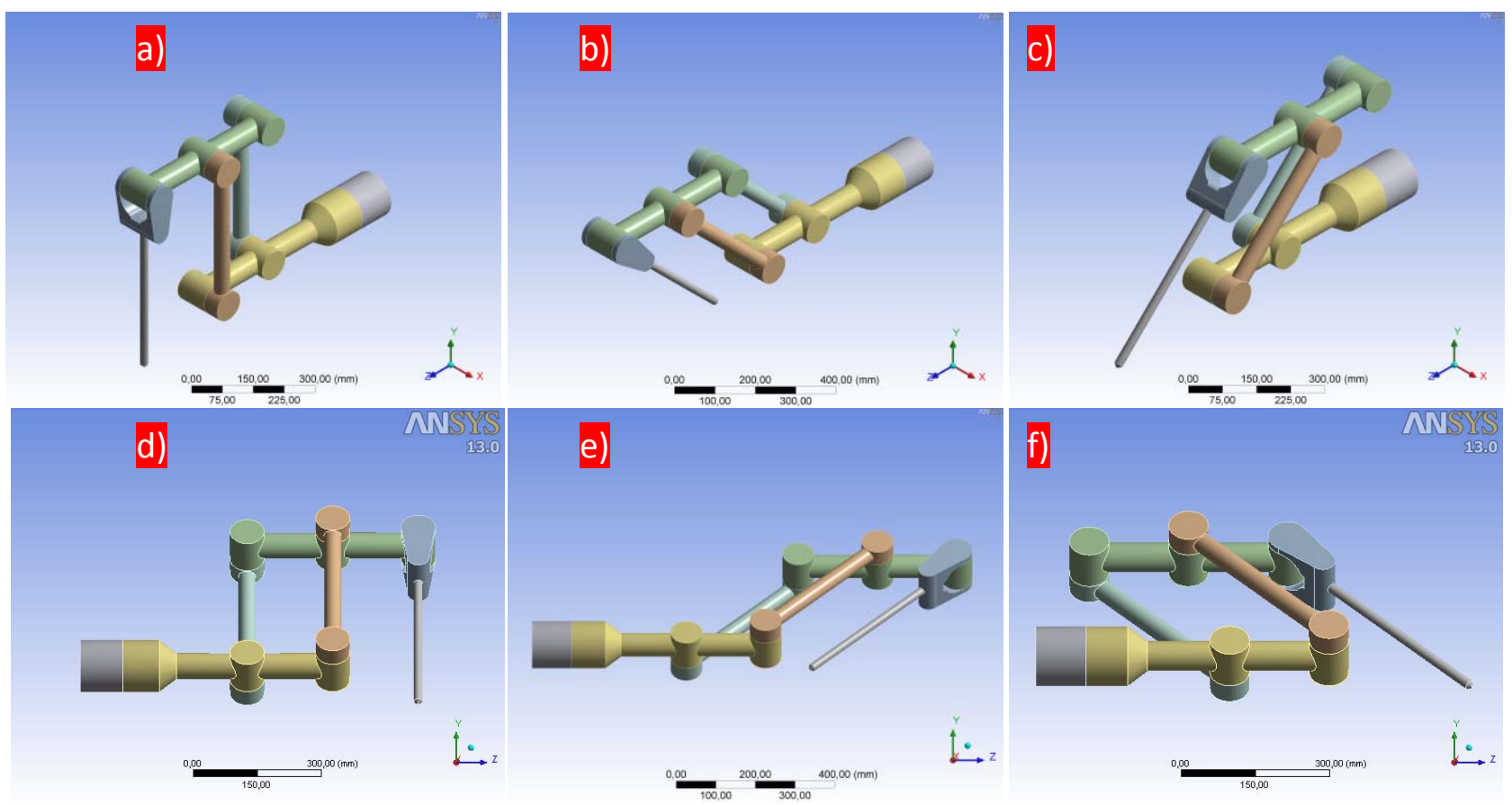

Fig.5. View of case study of telemanipulator in: a) Normal 90 degree; b) 45 degree; c) 135 degree, d) Normal 90 degree/45 side; e) 45 degree/45 side; f) 135 degree/45 side. 
In configuration II we used: 23881 nodes in case study 1; 24073 nodes in case study 2; 23932 nodes in case study 3; 23807 nodes in case study 4; 23999 nodes in case study 5; 24228 nodes in case study 6 and appropriately 10236 elements in case study 1; 10299 elements in case study 2; 10234 elements in case study 3; 10125 elements in case study 4; 10231 elements in case study 5; 10409 elements in case study 6 .

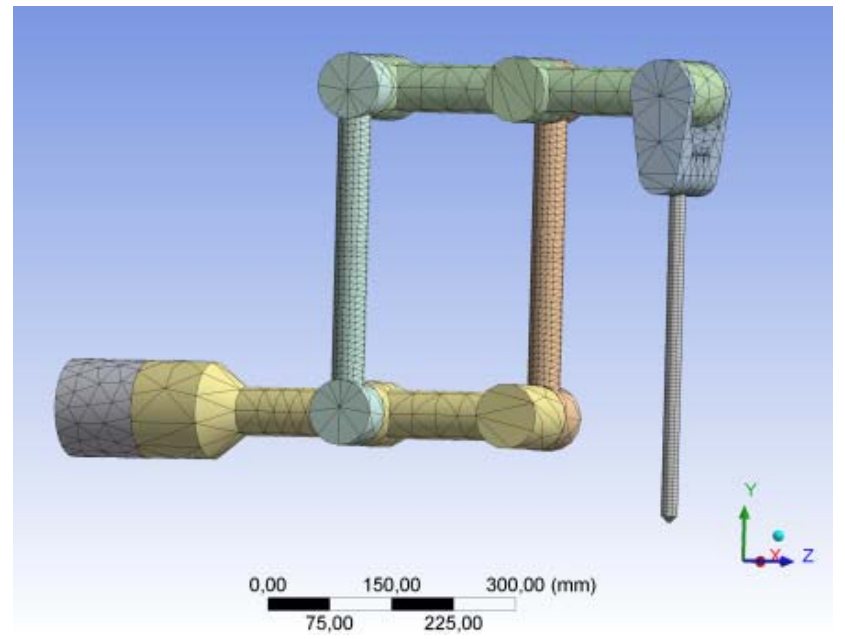

Fig.6. View of an example of mesh used in the analysis of the telemanipulator in configuration I.

In configuration III we used: 22012 nodes in case study $1 ; 22237$ nodes in case study 2; 22530 nodes in case study 3; 21921 nodes in case study 4; 21958 nodes in case study 5; 22367 nodes in case study 6 and appropriately 9505 elements in case study 1; 9574 elements in case study 2; 9693 elements in case study 3; 9368 elements in case study 4; 9412 elements in case study 5; 9666 elements in case study 6. In configuration IV used: 23088 nodes in case study 1; 23938 nodes in case study 2; 23177 nodes in case study 3; 22982 nodes in case study 4; 21958 nodes in case study 5; 22367 nodes in case study 6 and appropriately 10041 elements in case study 1; 10480 elements in case study 2; 10125 elements in case study 3; 10008 elements in case study 4; 9412 elements in case study 5; 9666 elements in case study 6. In configuration V used: 23615 nodes in case study 1; 24380 nodes in case study 2; 23825 nodes in case study 3;23684 nodes in case study 4; 24417 nodes in case study 5; 23614 nodes in case study 6 and appropriately 10274 elements in case study 1; 10683 elements in case study 2; 10460 elements in case study 3; 10349 elements in case study 4; 10726 elements in case study 5; 10320 elements in case study 6.

\section{Results of numerical analysis by the finite element method}

Table 4 and Table 5 contain the results of numerical simulations of static deformations of parallel manipulators' structures under the influence of $+50 \mathrm{~N}$ and $-50 \mathrm{~N}$ loads. Numerical simulations were performed in the ANSYS Workbench 13.0 software environment. The tables include data about absolute deformation of the entire structure under the action of applied load and directional deformation of the TCP system of the end effector on the XYZ axes, as well as the absolute value of deformation in the TCP system. Additionally they contain Max value of Equivalent (Huber von - Misses) Elastic Strain and Max value of Equivalent (Huber von - Misses) Stress. Fields with minimum values are green, and fields with maximum values are red. 
Table 4. Results of MES analysis in ANSYS - Force +50N.

\begin{tabular}{|c|c|c|c|c|c|}
\hline & Config. I & Config. II & Config. III & Config. IV & Config. V \\
\hline Case of study - Normal 90 degree & Force $+50 \mathrm{~N}$ & Force $+50 \mathrm{~N}$ & Force $+50 \mathrm{~N}$ & Force $+50 \mathrm{~N}$ & Force $+50 \mathrm{~N}$ \\
\hline Max value of Total deformation $[\mathrm{mm}]$ & 0.21327 & 0.19738 & 0.16306 & 0.25908 & 0.23347 \\
\hline Max of X - Axis Directional Deformation of TCP [mm] & 0.04787 & .03656 & -0.00424 & -0.05307 & -0.08984 \\
\hline xis Directional Deformation of TCP [mm] & 0.0806 & 0.05996 & 0.03413 & 0.08466 & 0.07915 \\
\hline Max of Z-Axis Directional Defon & -0.15327 & -0.14411 & -0.01202 & -0.16852 & -0.15391 \\
\hline Total deformation of TCP po & 0.17966 & 0.15966 & 0.12504 & 0.19598 & 0.19498 \\
\hline Max value of Equivalent (von - Misses) Elastic S & 0.00003 & 0.00003 & 0.00003 & 0.00004 & 0.00003 \\
\hline Max value of Equivalent (von - & 6.3928 & 6.9868 & 6.4337 & 8.063 & 7.1106 \\
\hline Case of study - 45 degree & Force $+50 \mathrm{~N}$ & Force $+50 \mathrm{~N}$ & Force $+50 \mathrm{~N}$ & Force $+50 \mathrm{~N}$ & Force $+50 \mathrm{~N}$ \\
\hline $\operatorname{Max}$ & 0.65975 & 0481 & 0296 & 0.67778 & 0.57705 \\
\hline of TCP [mm] & 0.03719 & -0.00322 & -0.00924 & -0.02281 & -0.03103 \\
\hline $\mathrm{fTCP}[\mathrm{mm}]$ & -0.33858 & -0.2257 & -0.25502 & -0.36955 & -0.38047 \\
\hline & & & 3266 & -0.56689 & -0.43214 \\
\hline & & & & 0.67695 & 0.5767 \\
\hline & 012 & 009 & 009 & 0.00041 & 0.00022 \\
\hline & 24.977 & 18.487 & 443 & 78.843 & 44.354 \\
\hline & $+50 \mathrm{~N}$ & $+50 \mathrm{~N}$ & $+50 \mathrm{~N}$ & Force $+50 \mathrm{~N}$ & Force $+50 \mathrm{~N}$ \\
\hline & 0.74258 & & 871 & 0.73079 & 0.71773 \\
\hline & 3702 & & & -0.00325 & -0.03184 \\
\hline & 0.46903 & & & 0.46098 & 0.4562 \\
\hline & & & & -0.56652 & -0.55367 \\
\hline & & & & 0.73037 & 0.71741 \\
\hline & 012 & 009 & 009 & 0.00012 & 0.00012 \\
\hline & 24.928 & 309 & 307 & 24.812 & 24.804 \\
\hline & Force $+50 \mathrm{~N}$ & $+50 \mathrm{~N}$ & $+50 \mathrm{~N}$ & Force $+50 \mathrm{~N}$ & Force $+50 \mathrm{~N}$ \\
\hline & 524 & & 346 & 0.66102 & 0.65663 \\
\hline & 863 & 039 & 127 & 0.34951 & 0.34583 \\
\hline & & & & 0.09033 & 0.08535 \\
\hline & & & 517 & -0.55292 & -0.55083 \\
\hline & & & & 0.66023 & 0.65552 \\
\hline & 013 & 0012 & 012 & 0.00014 & 0.00014 \\
\hline & 907 & .59 & 597 & 27.422 & 27.422 \\
\hline & $-50 \mathrm{~N}$ & $+50 \mathrm{~N}$ & $+50 \mathrm{~N}$ & Force $+50 \mathrm{~N}$ & Force $+50 \mathrm{~N}$ \\
\hline & & & & 0.83652 & 0.8462 \\
\hline & & & 904 & 0.20557 & 0.20649 \\
\hline & & & 724 & -0.24956 & -0.25114 \\
\hline & & & & -0.77094 & -0.78053 \\
\hline & & & & 0.8358 & 0.84557 \\
\hline & & & 013 & 0.00055 & 0.00055 \\
\hline & & .326 & 335 & 106.75 & 106.85 \\
\hline & $+50 \mathrm{~N}$ & $+50 \mathrm{~N}$ & $+50 \mathrm{~N}$ & Force $+50 \mathrm{~N}$ & Force $+50 \mathrm{~N}$ \\
\hline & & 0.63264 & 6491 & 0.8358 & 0.82637 \\
\hline & 0.16527 & -0.15106 & -0.12764 & 0.15703 & 0.15967 \\
\hline & 0.33633 & & 1732 & 0.33581 & 0.33322 \\
\hline Max of Z - Axis Directional Deformation of TCP [mm] & -0.75214 & & -0.50561 & -0.74939 & -0.73979 \\
\hline & & & & 0.8351 & 0.82572 \\
\hline & & & & 0.00016 & 0.00016 \\
\hline & & 24.268 & 24.267 & 31.719 & \\
\hline
\end{tabular}


Table 5. Results of MES analysis in ANSYS - Force $-50 N$.

\begin{tabular}{|c|c|c|c|c|c|}
\hline & Config. I & Config. II & Config. III & Config. IV & Config. V \\
\hline Case of study - Normal 90 degree & Force-50N & Force-50N & Force $-50 \mathrm{~N}$ & Force $-50 \mathrm{~N}$ & Force $-50 \mathrm{~N}$ \\
\hline Max value of Total deformation [mm] & 0.10415 & 0.10119 & 0.09063 & 0.13719 & 0.12338 \\
\hline Max of X - Axis Directional Deformation of TCP [mm] & .01301 & -0.00901 & -0.00147 & -0.01889 & -0.04021 \\
\hline Max of Y - Axis Directional Deformation of TCP [mm] & 0.02994 & 0.0245 & 0.01703 & 0.03708 & 0.0344 \\
\hline Max of Z - Axis Directional Deformation of TCP [mm] & -0.07661 & -0.07498 & -0.06705 & -0.09187 & -0.08271 \\
\hline Total deformation of TCP point [mm] & 0.08327 & 0.0794 & 0.069213 & 0.10089 & 0.09817 \\
\hline Max value of Equivalent (von - Misses) Elastic Strain & 0.00001 & 0.00002 & 0.00001 & 0.00002 & 0.00002 \\
\hline Max value of Equivalent (von- & 3.7779 & 3.9365 & 3.7967 & 5.0694 & 4.4551 \\
\hline Case of study - 45 degree & Force-50N & Force-50N & Force $-50 \mathrm{~N}$ & Force $-50 \mathrm{~N}$ & Force $-50 \mathrm{~N}$ \\
\hline Max value of Tc & 0.47729 & 0.33909 & 0.31709 & 0.44441 & 0.49634 \\
\hline nation of TCP [mm] & 0.03241 & -0.02572 & -0.00594 & -0.01636 & -0.02616 \\
\hline of TCP [mm] & 0.4158 & 0.30989 & 0.28148 & 0.38307 & 0.37333 \\
\hline f TCP [mm] & & 0.13194 & 0.144 & 0.223 & 0.32609 \\
\hline & & & 0.31611 & 0.4434 & 0.49608 \\
\hline Max & 0.0001 & 0.00008 & 0.00008 & 0.00036 & 0.00022 \\
\hline Max & 20.805 & 15.639 & 15.634 & 70.344 & 42.487 \\
\hline Cas & Force-50N & Force-50N & Force $-50 \mathrm{~N}$ & Force $-50 \mathrm{~N}$ & Force $-50 \mathrm{~N}$ \\
\hline $\operatorname{Max}$ & 0.52489 & 0.38299 & 36113 & 0.52016 & 0.5297 \\
\hline [mm] & -0.03236 & 2608 & 0.00244 & 0.03196 & -0.03946 \\
\hline & & -0.26398 & -0.25606 & -0.35909 & -0.3623 \\
\hline & & & & 0.37508 & 0.38445 \\
\hline & & & & 0.52006 & 0.52956 \\
\hline & 0.0001 & 0.00008 & 00008 & 0.0001 & 0.0001 \\
\hline $\mathrm{Ma}$ & 20.676 & 15.485 & 15.483 & 20.535 & 20.538 \\
\hline Cas & Force-50N & Force-50N & Force $-50 \mathrm{~N}$ & Force $-50 \mathrm{~N}$ & Force $-50 \mathrm{~N}$ \\
\hline & 0.53771 & 798 & 5893 & 0.54432 & 0.55191 \\
\hline & 0.44032 & 7472 & 8441 & -0.45307 & -0.45841 \\
\hline & & 497 & 323 & 0.07831 & 0.0815 \\
\hline & & & & 0.2911 & 0.29472 \\
\hline & & & & 0.54313 & 0.55073 \\
\hline & 0.00011 & 0.00011 & 0011 & 0.00011 & 0.00011 \\
\hline & 22.24 & 20.815 & 20.797 & 22.616 & 22.613 \\
\hline & $-50 \mathrm{~N}$ & Force-50N & e-50N & Force $-50 \mathrm{~N}$ & Force $-50 \mathrm{~N}$ \\
\hline & 0.59596 & 7016 & 1417 & 0.53381 & 0.5311 \\
\hline & -0.22555 & 0.13825 & 728 & -0.19071 & -0.19069 \\
\hline & 0.32854 & & & 0.28621 & 0.28518 \\
\hline & & & & 0.40708 & 0.404 \\
\hline & & & & 0.53302 & 0.53086 \\
\hline & 0.00011 & 0.00011 & 0.00011 & 0.00059 & 0.00061 \\
\hline Ma & 22.149 & 21.481 & 21.467 & 115.57 & 117.59 \\
\hline & $-50 \mathrm{~N}$ & Force-50N & Force $-50 \mathrm{~N}$ & Force $-50 \mathrm{~N}$ & Force $-50 \mathrm{~N}$ \\
\hline & 0.59743 & 0.5131 & 0.44278 & 0.59235 & 0.59556 \\
\hline & -0.21099 & 0.12346 & 0.12556 & -0.21822 & -0.21985 \\
\hline & -0.23097 & & -0.18117 & -0.22653 & -0.22533 \\
\hline Max of Z - Axis Directional Deformation of TCP [mm] & 0.50876 & & 0.38434 & 0.50167 & 0.5047 \\
\hline & & & & 0.59189 & 0.59518 \\
\hline & & & & 0.00013 & 0.00013 \\
\hline Max value of Equivalent (von - Misses) Stress [MPa] & 26.234 & 20.533 & 20.524 & 26.338 & 26.348 \\
\hline
\end{tabular}


The results will serve for selection of the most favorable confirmations. The following will be taken as selection criteria: weight of the system, stresses present in the structure, and rigidity, which translates to the precision of a surgical instrument's movement. Considering the weight of the entire manipulator due to its having the lowest number members in the mechanism, the weight of the system in configuration I is the lowest and is equal to $51.783 \mathrm{~kg}$. In this case, however, we are dealing with an active remote center of motion, therefore, additional encoders and drives required for controlling the system may increase the manipulator's weight in reality. The addition of an extra member allowing to realization of a kinematic RCM to the manipulator's kinematic structure is the most favorable in the case of configuration III - here, the weight is 52.1 $\mathrm{kg}$. The system's lower weight will translate to a reduction of the influence of forces of inertia, which have a negative effect on the system's operation during a surgical procedure, thus configuration III is the best choice.

An analysis of the results of static displacements of mechanisms in the five configurations indicated above, for the six adopted cases of member positions, clearly shows that the best results are obtained in the case where the manipulator is in configurations III and II. Configuration I has the worst results, followed by configuration IV and V.

In the case where the parallelogram mechanism of the manipulator is set in a position in which all of the paralleolgram's links are positioned at an angle of 90 degrees relative to one another and the force is equal to $-50 \mathrm{~N}$, deformation of TCP point position is approx. $0.07 \mathrm{~mm}$ (see Tab.5), and this is the minimum value for all of the cases under analysis (see Fig.7a). The value of deformation at point TCP amounts to approx. $0.1 \mathrm{~mm}$, being more than $30 \%$ greater than the minimum value, when the mechanism's members are positioned and forces act as in configuration IV. In configuration III, when the loading direction is changed $(+50 \mathrm{~N})$, TCP deformation values nearly double, reaching up to approx. $0.12 \mathrm{~mm}$.

The case of the robot in configuration $\mathrm{V}$, under $+50 \mathrm{~N}$ load in the position in which the mechanism's links are inclined at an angle of 45 degrees relative to the lower main link and also axially inclined at an angle of 45 degrees relative to the first link, is the least favorable. The absolute deformation of point TCP reaches its maximum in this configuration, reaching up to approx. $0.85 \mathrm{~mm}$ (see Tab.4 and Fig.7b), which, in comparison to the case of configuration III - with the best results in the same position (deformation value is approx. 0.56 $\mathrm{mm}$ ), is rather significant for the assessment of positioning accuracy.

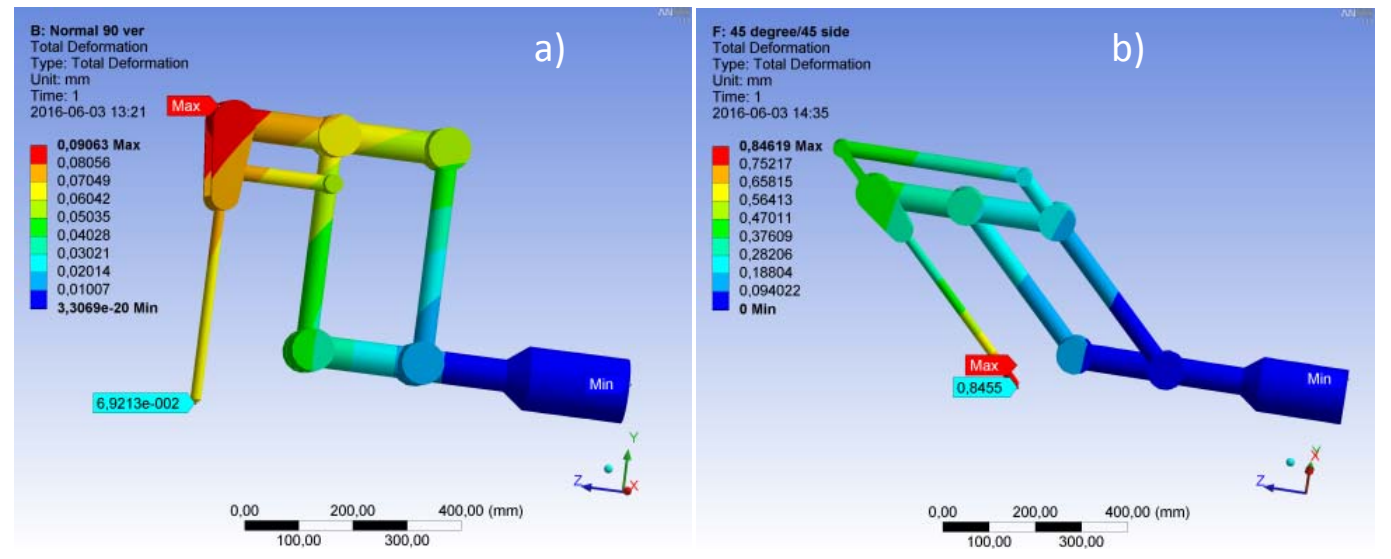

Fig.7. View of case study of telemanipulators: a) configuration III (Normal 90 degree) - best result; b) configuration V (45 degree/45 side) - worst result.

Considering the maximum value of equivalent (von - Misses) elastic strain in the cases, the greatest values were obtained for configuration $\mathrm{V}-0.00061$ (see Tab.5) in the case where the manipulator is inclined relative to two planes at an angle of 45 degrees (case study 45 degree/45 side). However, when one accounts for the maximum value of equivalent (von - Misses) stress, the lowest values occur in cases where the parallelogram mechanism remains in the Normal 90 degree position and is subjected to a force of $-50 N$. The greatest stress values are observed in the structures in the case of the 45 degree manipulator position, and these stresses reach up to $78.843 \mathrm{MPa}$ in the case of configuration IV. 


\section{Conclusions}

- Configuration III, and potentially II, is the most favorable among the studied structures of surgical manipulators with a parallel manipulation mechanism for kinematic preservation of the remote center of motion for the instrument. Despite having apparently lower weight of its members, the classical parallelogram imposes the application of additional mechatronic equipment in order to enable preservation of the remote center of motion, and this will increase weight, inertia, and affect positioning accuracy directly.

- The quality of positioning of a surgical robotic system depends on stiffness of the telemanipulator. Positioning errors in an actual telemanipulator configuration should be taken into account in the control algorithm. The results of numerical simulation should be applied in this algorithm.

- Stiffness of the structure is a very important factor to be considered when designing surgery telemanipulators which will provide accurate positioning of a surgical instrument.

This work was performed within the framework of statutory research of the Department of Automatic Control and Robotics at Bialystok University of Technology no. S/WM/1/2016 and financed with funds from the Ministry of Science and Higher Education.

\section{Nomenclature}

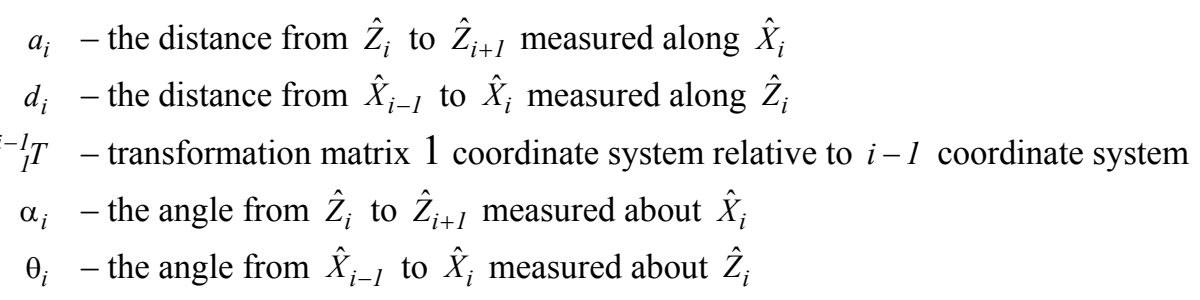

\section{References}

[1] Podsędkowski L. (2010): Medical robotic: Construction and Applications. - Warsaw: WNT (in Polish).

[2] Kroczek P. and Cieślik J. (2013): Analysis of kinematic structure of RobIn Heart Vision robot In: Postępy inżynierii biomedycznej (L. Leniowska, Z. Nawrat, Ed.). - Rzeszow University, pp.331-338 (in Polish).

[3] Podsędkowski L., Mianowski K., Wróblewski P. and Nawrat Z. (2002): Kinematic aspects of selected manipulators for cardiac surgery. - On IEEE International Conference of Methods and Models in Automation and Robotics, pp.923-928.

[4] Trochimczuk R. (2013): Conception of arm of medical robot dedicated to application of minimally invasive surgery. - Solid State Phenomena, vol. 198, Trans Tech Publications, pp.3-8.

[5] Trochimczuk R., Łukaszewicz A., Mikołajczyk T., Aggogeri F. and Borboni A. (2016): Stiffness FEM analysis of novel telemanipulator for minimally invasive surgery. - Journal of Intelligent \& Robotic Systems (in review process).

[6] Kuo C.H., Dai J.S. and Dasgupta P. (2012): Kinematic design considerations for minimally invasive surgical robots: an overview. - The International Journal of Medical Robotics and Computer Assisted Surgery, vol.8, No.2, Willey Online Library, pp.127-145.

[7] Li J., Zhang G,, Xing Y., Liu H. and Wang S. (2014): A Class of 2-Degree-of-Freedom Planar Remote Center-ofMotion Mechanisms Based on Virtual Parallelograms. - ASME. Journal of Mechanisms and Robotics, vol.6, No.3, 031014, 08.2014.

[8] Craig J.J. (1995): Introduction to Robotics. Mechanics and control. - Warsaw: WNT (in Polish). 